\title{
Estado del arte sobre problemáticas financieras en pymes: estudio para América Latina
}

\author{
Sandra Yadira Laitón Ángel ${ }^{1}$ \\ Universidad de La Salle \\ slaiton33@unisalle.edu.co \\ Juanita López Lozano² \\ Universidad de La Salle \\ juanitalopez15@unisalle.edu.co
}

DOI: https://doi.org/10.21158/01208160.n85.2018.2056

Fecha de recepción: 27 de febrero de 2018

Fecha de aprobación: 01 de agosto de 2018

Cómo citar este artículo / To reference this article / Comment citer cet article / Para citar este artigo:

Laitón Ángel, S Y. y López Lozano, J. (2018). Estado del arte sobre problemáticas financieras en pymes: estudio para América Latina. Revista EAN, 85, 163-179. DOI: https://doi.org/10.21158/01208160.n85.2018.2056

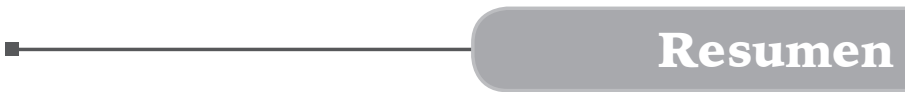

El artículo presenta los resultados de la revisión bibliográfica realizada con el objetivo de identificar las principales problemáticas financieras que afrontan las pequeñas y medianas empresas de algunos países latinoamericanos. En este sentido, se llevó a cabo una investigación de tipo cualitativo con un alcance descriptivo, en la que a partir del análisis de la información encontrada en la literatura, se construyeron tres núcleos problemáticos: estructura de capital, acceso a la financiación y gestión de la planeación estratégica. Se evidenció que un gran porcentaje de estas pymes presentan similitudes en la forma en que administran sus recursos, la manera en la que toman las decisiones de financiación y los desafíos a los que se enfrentan a diario. Uno de los elementos que se consideró con detalle fue el nivel de educación del gerente de la compañía, dado que puede entenderse como un factor relevante a la hora de aprovechar las ventajas de las herramientas financieras y propiciar el crecimiento de las empresas.

\section{Palabras clave}

Pequeñas y medianas empresas - aspectos económicos, planeación estratégica, financiamiento de empresas, administración de recursos, estado del arte, revisión de la literatura.

${ }^{1}$ Estudiante de décimo semestre del programa de Finanzas y Comercio Internacional de la Universidad de La Salle, perteneciente al semillero de investigación SIAFCI.

2 Estudiante de décimo semestre del programa de Finanzas y Comercio Internacional de la Universidad de La Salle, perteneciente al semillero de investigación SIAFCI. 


\title{
State of the art on financial problems in pymes: a Latin American study
}

\begin{abstract}
This article describes the outcomes of a bibliographic revision made to identify the main financial problems that small and medium- size companies face in some Latin-American countries. For this reason, a qualitative descriptive research study is made, from which three problematic areas are defined: capital structure, financing access and strategic planning management. It was found that a high percentage of these pymes have similarities in the way they manage their resources, the way they make financial decisions and the daily problems they face. One of these issues is the educational level of the company chief executive because it turns to be the most relevant factor at the time of taking advantage from financial tools and allowing company growth.
\end{abstract}

Key words. Pymes- small and medium- size companies- economic aspects, strategic planning, company financing, resource management, state of the art, bibliographic revisión.

\section{Analyse des problématiques financières des PME: une étude pour l'Amérique latine}

Résumé. L'article présente les résultats d'une analyse bibliographique réalisée dans le but d'identifier les principales problématiques financières rencontrées par les petites et moyennes entreprises dans certains pays d'Amérique latine. Une recherche qualitative à portée descriptive a alors été réalisée. L'analyse des informations recensées identifie trois types de problématiques: la structure du capital, l'accès au financement et la gestion de la planification stratégique. L'étude révèle qu'un fort pourcentage de PME présente des similitudes dans le mode de gestion des ressources financières, dans les prises les décisions de financement et dans les défis auxquels elles se trouvent confrontées au quotidien. Un éléments ayant été examiné avec attention se trouve être le niveau de formation du dirigeant de l'entreprise, facteur d'importance permettant de tirer au mieux parti des outils financiers et de promouvoir la croissance des entreprises.

Mots clefs. Petites et moyennes entreprises - aspect économique, planification stratégique, financement des entreprises, gestion des ressources, analyse des sources d'informations.

\section{Estado da arte sobre problemáticas financeiras em PMEs: estudo para a América Latina}

Resumo. O artigo apresenta os resultados da revisão bibliográfica realizada como objetivo de identificar as principais problemáticas financeiras que enfrentam as pequenas e médias empresas de alguns países latino-americanos. Neste sentido, realizou-se uma pesquisa de tipo qualitativo com um alcance descritivo, na qual a partir da análise da informação encontrada na literatura, se construíram três núcleos problemáticos: estrutura de capital, acesso ao financiamento e gerenciamento do planejamento estratégico. Evidenciou-se que uma grande porcentagem destas PMEs apresenta semelhanças na forma em que administram seus recursos, na maneira como tomam as decisões de financiamento e nos desafios que enfrentam diariamente. Um dos elementos que se considerou com detalhe foi o nivel de educação do gerente da companhia, dado que pode ser entendido como um fator relevante na hora de aproveitar as vantagens das ferramentas financeiras e propiciar o crescimento das empresas.

Palavras-chave. Pequenas e médias empresas - aspectos econômicos; planejamento estratégico; financiamento de empresas; administração de recursos; estado da arte; revisão da literatura. 


\section{Introducción}

L as pequeñas y medianas empresas (pymes), representan una fuerza económica para los países latinoamericanos, dado que constituyen al menos el $85 \%$ del tejido empresarial y generan alrededor del $50 \%$ de los empleos (Raufflet, Lozano, Barrera, García, 2012). No obstante, se debe considerar que cada país cuenta con metodologías diferentes para definir el concepto de pyme. Guatemala, Brasil y Costa Rica clasifican las empresas de acuerdo con el número de empleados, Perú y Chile las agrupan de acuerdo con el nivel de ventas y de empleados, Colombia a partir del valor de los activos y de la cantidad de empleados, Argentina y Honduras conforme el volumen de ventas, y México según número de empleados y sector (Chiatchoua y Castañeda, 2015; Raufflet et al., 2012; Zevallos, 2003).

Ahora bien, se ha evidenciado que este tipo de empresas no logra permanecer en el mercado por mucho tiempo, debido a sus altas tasas de mortalidad, en las que el $80 \%$ de las pymes no logra superar los dos años (Raufflet et al., 2012); ya que en el desarrollo de sus actividades enfrentan diferentes retos, para los cuales no muchas están preparadas $y$, por tanto, se ocasiona el cierre de estas, trayendo consigo una escasez de empleos y un declive en la economía.

Una de las principales razones por las que se presenta el cierre de estas empresas, corresponde a la imposibilidad de acceder a los recursos, tanto financieros como intelectuales, que requiere la pyme para su funcionamiento, y a la incapacidad de sus directivos para gestionarlos de forma exitosa (Vera, Melgarejo y Mora, 2014). Es por esto que el problema de financiamiento toma gran relevancia para las pymes en Latinoamérica, pues sin el acceso a fuentes de financiación y sin flujos suficientes, la empresa no puede operar y tiene menos posibilidades para sobrevivir (Saavedra y Leticia, 2014).

Debido a esto, las pymes buscarán la forma más adecuada de apalancamiento, la cual se relaciona con la teoría del Pecking Order desarrollada por Myers y Majluf (1984), en la que se establece una jerarquía con respecto a las decisiones de financiación, teniendo preferencia por el financiamiento interno, seguido de la deuda y por último el aumento de capital. Así mismo, la teoría de Trade Off propuesta por Kraus y Litzenberger (1973), afirma que las empresas buscan la mejor relación deuda/ patrimonio, a partir de un equilibrio entre las desventajas derivadas de las probabilidades de quiebra y las ventajas tributarias de la deuda.

A partir de esto surge la necesidad de documentar las principales problemáticas financieras de las pymes en América Latina para mantenerse en el mercado, y así recopilar información para que en estudios futuros se tenga un amplio marco de referencia sobre los factores que obstaculizan el ciclo de vida de este segmento de empresas. No obstante, en la revisión documental realizada, se identifican las problemáticas por separado, teniendo en cuenta que no hay un artículo actualizado que consolide todos los factores.

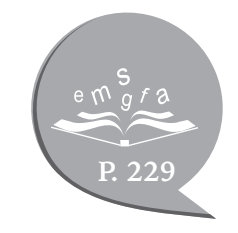


Por último, el objetivo es identificar, a partir de la revisión de literatura, las principales problemáticas financieras que tienen las pymes de algunos países latinoamericanos, para mantenerse en el mercado. Por lo anterior, se organizó el artículo de la siguiente manera: en la primera parte se describe la metodología utilizada para la generación del documento. En la segunda se presentan los hallazgos de la investigación separados en 3 núcleos problemáticos: estructura de capital, acceso a la financiación y planeación estratégica. Por último, se presentan las conclusiones del análisis.

\section{Metodología}

$\mathbf{P}$ ara el desarrollo del presente artículo se elaboró una investigación de tipo cualitativa con un alcance descriptivo, basada en la revisión de artículos científicos publicados en el periodo 2013-2017, en algunas bases de datos como: Elsevier, Springer, Google Académico y ScienceDirect; cuyo enfoque estuviera dirigido a las problemáticas financieras de las pymes de algunos países latinoamericanos, que para el caso fueron: Chile, Costa Rica, Argentina, Colombia, México, Ecuador y Perú. Para obtener los documentos que cumplieran con las características mencionadas, se filtró la búsqueda hacia aquellos documentos cuyas palabras clave -tanto en inglés como en españolestuvieran relacionadas con la temática de investigación, tales como: pymes, problemáticas financieras, fracaso empresarial, entre otras.
Una vez hecho esto, se dio paso a la identificación de los elementos representativos en cada uno de los artículos revisados, elaborando una lista con 20 factores financieros que incidían de forma negativa en el desarrollo de los objetivos de las empresas. Posterior a ello, se realizó el tratamiento de la información mediante un contraste comparativo por medio de la creación de matrices que permitieran identificar los temas comunes, para así clasificar y sintetizar los factores previamente analizados en tres grandes núcleos problemáticos, definidos como: estructura de capital, acceso a la financiación y gestión de la planeación estratégica.

Por último, con base en los núcleos problemáticos nombrados anteriormente, se dio lugar a la redacción del estado del arte, a partir de la consolidación de los hallazgos o ideas más relevantes de cada escrito. 


\section{Hallazgos}

$\triangle$ partir de la metodología descrita, los 3 de la revisión se desarrollan a continuación:

\subsection{Estructura de capital}

«La jerarquía y las teorías de costo de agencia sobre la estructura de capital, explican en parte el comportamiento financiero de las pymes» (Xiang y Worthington, 2015, p. 515). La estructura de capital representa un papel

Figura 1. Estructura de capital relevante al momento de analizar la situación financiera de las pequeñas y medianas empresas. Como se muestra en la figura 1, Blasco, Guaita y Postiguillo (2016) identifican que dentro del análisis de la estructura de capital, un menor costo de capital conlleva a un mayor valor de la compañía. Es relevante tener en cuenta que la determinación de una estructura de capital óptima, tiene mayor influencia en la empresa que efectos industriales o institucionales (Macan Bhairda y Lucey, 2014).

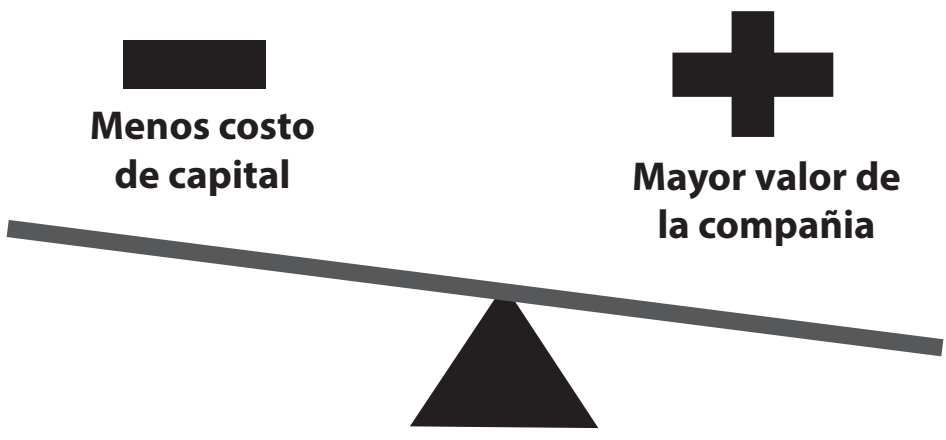

Fuente. Elaboración propia, adaptado de Blasco et al., 2016.

Pavón (2016) encuentra como una de las limitaciones de la estructura de capital, que propietarios de las pequeñas y medianas empresas no quieren asumir riesgos relacionados a préstamos bancarios. En el caso de las pymes mexicanas, se cuenta con un orden jerárquico para la toma de decisiones, pues estas anteponen el uso de recursos propios como primera opción frente a la financiación externa (Duarte, Pastrana y Rosado, 2015). Similar a ello, el microempresario de Lima, Perú, «prefiere el manejo de los fondos de su negocio conjuntamente con sus ingresos y gastos personales» (Alva, 2017, p. 87).
Para las pymes del sector comercial de Machala, Ecuador, los préstamos bancarios son el principal método de financiamiento para el $72 \%$ de las pymes encuestadas (Córdova, Montalvo y Bohórquez, 2014). Sin embargo, Capa, Capa y Ollague, (2018), mencionan que la principal fuente de financiación de las pymes ecuatorianas es la otorgada por los proveedores, pues estos representan el $42 \%$ del total de las fuentes de financiación disponibles.

Contrario a lo anterior, en el estudio realizado en Chile, las pymes tienen una inclinación al modelo de Trade Off, dejando de lado la 
opción de autofinanciamiento (Gutiérrez, Aguayo y Panes, 2014). De acuerdo con Brenes y Bermúdez (2013), en Costa Rica los proveedores representan una alternativa relativamente importante para las pymes del sector industrial y el de servicios; mientras que para las de agricultura y pesca los préstamos personales son utilizados en un alto porcentaje.

Ruiz (2015) indica que la banca comercial es tomada por las pymes como principal fuente de financiación; este apalancamiento en América Latina, específicamente en Colombia, se encuentra en niveles que superan el $50 \%$ de la deuda, y el costo de financiación equivale en promedio al $86 \%$ del margen operacional, dejando como margen de utilidad neto del 3 $\%$ al $5 \%$; este valor final no logra cumplir con las expectativas de los propietarios, los cuales esperan una rentabilidad final que oscile entre el $5 \%$ al $10 \%$. Vera et al., (2014) respaldan esta afirmación y añaden que dichos porcentajes de utilidad neta, igualan o superan por muy poco los niveles de inflación de Colombia.

Por otra parte, para Xiang y Worthington (2015), existe una relación positiva entre el tamaño y la edad de la empresa, en el que las pymes en fase inicial tienen mayores probabilidades de buscar financiación interna que financiación bancaria. Según Kantis, Federico, Angelelli e Ibarra (2016) la mayor parte de la financiación de las empresas jóvenes latinoamericanas proviene de los ahorros personales o de dinero prestado de sus familiares y amigos; tal como plantean Prohorovs, Jakusonoka y Beizitere (2015).

Luego, a falta de capital los empresarios emprendedores, lo compensan con préstamos solicitados a personas naturales; sin embargo, la cantidad de efectivo necesaria para acelerar los procesos de crecimiento por lo general exceden esas fuentes personales; de esta manera, cuando la empresa comienza a crecer, los directivos deciden financiarse con instituciones de capital de riesgo y luego en el mercado de deuda (Guercio, Martínez y Vigier, 2017). Por lo que las proporciones de deuda y patrimonio, muestran cambios significativos dependiendo de la estabilidad de la pyme dentro del mercado.

Daskalakis, Eriotis, Thanou y Vasiliou (2014), en su estudio encontraron que la ratio de endeudamiento de las pymes está relacionado de forma negativa con la rentabilidad. Adicionalmente, si la empresa tiene una alta capacidad para generar fondos internos, dicha empresa tenderá a evitar el financiamiento de deuda, al igual que las empresas que dependen en alto grado de sus activos tangibles. Así mismo, el Banco Mundial (2014) propone que, para financiar el capital de trabajo, la mayoría de pymes se apoyan a partir de los recursos internos, de créditos con sus proveedores o de productos financieros como el factoring.

Para determinar problemáticas en la estructura de capital de pymes en Colombia, Vera et al. (2014) analizan algunos indicadores financieros en los que se evidencia que con respecto a la financiación de terceros, el endeudamiento con instituciones financieras representa el 9 $\%$ de los activos y, comparado con el nivel de ingresos, representa 14.6 veces el volumen de ventas; por lo tanto, estas empresas no estarían en condiciones de apalancarse con recursos bancarios, razón por la que acuden al endeudamiento patrimonial.

Otro de los aportes de estos autores radica en que la liquidez y la rotación pueden afectar negativamente la rentabilidad de la empresa, debido a una «alta liquidez, representada por deudas de clientes, baja rotación de cobranzas y recuperación de ingresos, frente a una rápida frecuencia de pago a proveedores" (Vera et al., 2014, p. 156). Sumado a esto, evidencian 
que los índices de solvencia son inadecuados, debido a que estos muestran que se tiene menor capacidad para cancelar sus deudas a largo plazo; lo cual puede conllevar a que surjan problemas a la hora de hacer frente a sus obligaciones con terceros, especialmente para el caso de pymes de cooperativas de trabajo asociado (Melgarejo, Vera y Mora, 2014).

Para el caso de Ecuador, Capa et al. (2018), encuentran que el $64 \%$ de las pymes evaluadas, no cuentan con una estrategia para determinar la estructura de capital adecuada, que garantice una estabilidad financiera y que responda a sus necesidades. Así mismo, las pymes manufactureras de Ecuador, toman sus decisiones de apalancamiento con base a las necesidades de rentabilidad, sobre la búsqueda de una mejor estructura de financiamiento, con el fin de maximizar el valor de la empresa (Espejo, Robles y Higuerey, 2017).

En la figura 2, se presenta un comparativo de las preferencias de financiación para el capital de trabajo, según el tipo de empresa y el país; en esta se evidencia que los fondos internos, a través de las utilidades retenidas, representan la primera opción para las pymes, seguido de las compras de crédito a proveedores y los préstamos bancarios, tanto públicos como privados. Así mismo, se evidencia que los préstamos de instituciones financieras no bancarias, representan la última opción como fuente de financiación para los países señalados en la figura (OCDE-ECLAC, 2012).

Figura 2. Comparación fuentes de financiación para el capital de trabajo

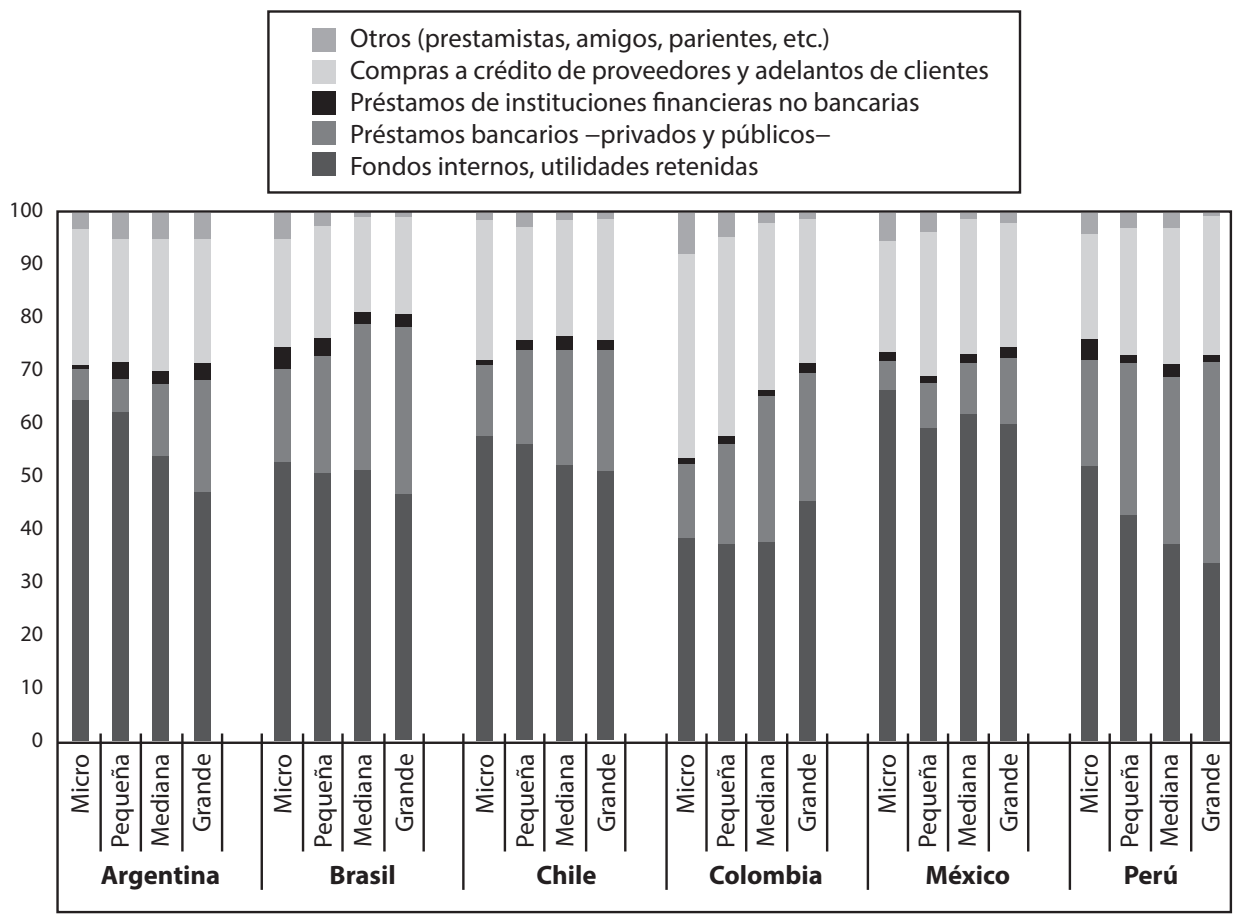

Fuente. OCDE-ECLAC, 2012. 


\subsection{Acceso a la financiación}

El Banco mundial (2014) y Dabla et al. (2015) plantean que las pymes suelen enfrentar limitaciones más fuertes al crecimiento que las grandes empresas, ya que tienen varias restricciones: el acceso a las finanzas, dado por los requerimientos de garantía; y el costo del crédito principalmente por elevadas tasas de interés, se identifican entre las más importantes. Comparado con otras regiones, en América Latina es baja la proporción de empresas que pueden financiarse a través del mercado de crédito (Botello, 2015).

Según Avilés (2017), la limitación al financiamiento bancario en las primeras etapas de una pyme causará que cualquier emprendimiento se vea afectado, ya que el resultado serán mayores costos para financiar activos requeridos en etapas tempranas y la inversión no se verá reflejada en el incremento del valor de la compañía. Con respecto a esto, las empresas peruanas presentan mayores desafíos con respecto al acceso a la financiación, dado que el sistema financiero no está completamente desarrollado y las facilidades bancarias son menores en comparación a otras economías (Duréndez y Rocca, 2017).

En América Latina y el Caribe, " $40 \%$ de las pequeñas empresas tiene acceso al financiamiento en el sistema financiero formal» (Pavón, 2016, p. 9). Teniendo en cuenta la estructura de capital típica en América Latina, una de las razones que hacen que las pymes no accedan en primera medida a la financiación externa, son las altas tasas de interés (Saavedra y Leticia, 2014). Sin embargo, Saavedra y Tapia (2013), refutan esta hipótesis explicando que cuando las empresas cuentan con altos niveles de rentabilidad, la tasa de interés es irrelevante.
Para el $78 \%$ de las pymes del sector comercial de Machala, Ecuador, el principal obstáculo al momento de buscar financiamiento son las altas tasas de interés (Córdova et al., 2014). Por otro lado, para el caso de Argentina y Brasil, la banca pública, en comparación con la banca privada, muestra una mayor financiación a empresas pequeñas, aquellas que cuentan con menor capacidad de activos y que están ubicadas en regiones socioeconómicas más pobres (Briozzo, Fernández, Villar y Pesce, 2016).

En otro caso, los gerentes prefieren mantener la información de la empresa de manera privada, por lo que utilizar sus fondos propios evita proporcionar al mercado dicha información (Briozzo, Vigier, Castillo, Pesce y Speroni, 2016). Además de esto, Becicova (2015) agrega que las personas sienten incertidumbre sobre el futuro de sus empresas y preocupación por no poder pagar el crédito.

Además de esto, otra de las barreras de las pymes para acceder a los créditos bancarios, son las garantías exigidas por los bancos. Como se evidencia en la figura 3, en América Latina el $72.4 \%$ de los créditos otorgados por los bancos a las empresas exige algún tipo de garantía; por su parte, en Colombia corresponde al $60.5 \%$, en Costa Rica este requisito alcanza el $97.2 \%$ de los créditos y en México al 67 \% (Lecuona, 2014). Debido a esto, muchos gerentes utilizan garantías personales sobre créditos tomados a nombre de la empresa o utilizan créditos personales para financiar la actividad de esta, dado que las limitaciones y requerimientos de dichos créditos son menores (Pesce, Esandi, Briozzo y Vigier, 2015). 
Figura 3. Porcentaje de bancos que exigen garantías

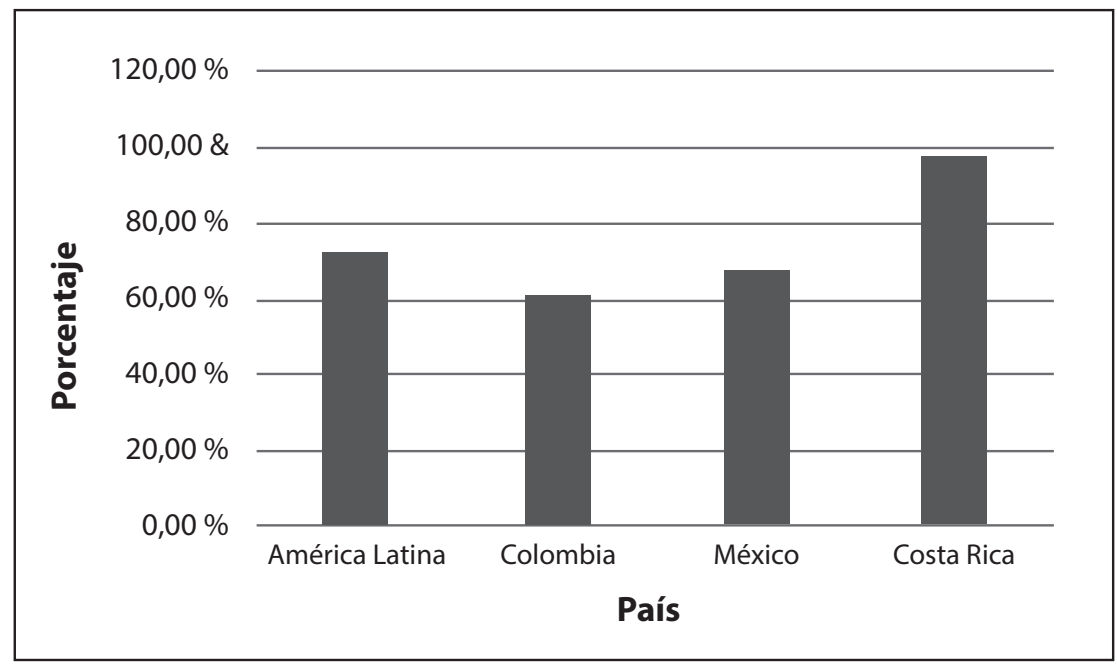

Fuente. Adaptado de Lecuona, 2014.

Osano y Languitone (2016), agregan que dichas garantías personales desincentivan a las pymes a adquirir financiamiento con los bancos, además de que son discriminadas por estos debido a los altos riesgos en los que incurren al ofrecer este tipo de préstamos. Esto puede llevar a que la contabilidad no refleje verdaderamente el estado de la empresa, pueden haber confusiones en el pago de los créditos y que exista la posibilidad de presentar problemas legales a futuro. Además, la mayoría de las pymes no producen estados financieros auditados que proporcionen información financiera creíble (OECD, 2015).

Sumado a esto, la información juega un papel importante al momento de tomar la decisión de financiación tanto para los bancos como para las empresas. Brancati (2015) afirma que, para los bancos, la cantidad de información recopilada sobre la solvencia de las empresas puede ser un factor crítico para determinar el acceso al crédito, especialmente para las pymes, cuya información no es suficiente. «Las pymes, en la mayoría de los casos, no cuentan con información financiera proyectada, carecen de hojas de balance general, estados de resultados y estado de costos de producción y ventas« (Blancas y López, 2014, p. 550).

Esto hace que exista un mayor riesgo moral, asociado a la probabilidad de que las empresas no actúen de manera consistente con la estipulación del contrato y al final no logren cancelar las deudas que tienen con las instituciones financieras (Motta, 2017). Además, Abor (2014) indica que «la mala calidad de los proyectos potenciales que califican para financiamiento y la incapacidad de las pymes para elaborar planes empresariales convincentes y estados financieros pro forma» (p. 98), son otras de las razones que impiden a los bancos financiar este tipo de empresas.

Mora y Castillo (2014) sustentan lo mencionado anteriormente dado que los bancos en Colombia solicitan el registro mercantil, el cual acredita la actividad comercial de la empresa, y también los estados financieros como requisito para otorgar créditos; debido a que muchas empresas no se encuentran registradas ante la Cámara de Comercio y en algunos casos no hay veracidad de los estados financieros, 
los bancos aplican altas tasas de interés o no conceden el crédito. Como consecuencia de esto, las pymes buscan financiación a través de créditos informales. Así mismo, en Guatemala, Nicaragua y Paraguay, el crédito informal juega un papel importante en el financiamiento de la compañía informal (Figueroa, 2014).

Para Jinjarak y Wingnaraja (2016), mejorar las alternativas de financiación y el apoyo del gobierno nacional derivan en uno de los mayores problemas para las pymes en mercados emergentes. Pavón (2016) explica que el limitado acceso al financiamiento no solo implica características propias de las pymes, sino también del sistema financiero local, en el que América Latina muestra un comportamiento más lento frente a países de la OCDE. Además, menciona que «en Ecuador, del total de crédito otorgado por el sistema financiero privado, $9.85 \%$ fue a pymes, $y$ en México solo $8.5 \%$ de empresas de ese segmento cuenta con un crédito y únicamente absorbieron $22 \%$ del financiamiento bancario empresarial» (p. 60).

Con respecto a Colombia, un estudio revela que, a pesar de que se han fijado políticas macroeconómicas para facilitar el acceso a créditos, los empresarios no conocen dichas oportunidades, por lo cual optan, en algunos casos, a la banca comercial como fuente de financiación externa (Ruiz, 2015). McGuinness y Hogan (2016), afirman que cualquier restricción al acceso al financiamiento de las grandes empresas aplicada por el gobierno, afectará negativamente a las pymes en este mismo segmento.

Por otro lado, el sector en el que se encuentra la pyme infiere en el acceso a financiación, como lo especifica Domeher, Musah, y Hassan, «la magnitud del déficit de financiación de las pymes varía según los sectores» (2017, p. 23). Además, estudios han comprobado que existen mayores limitaciones para las empresas que pertenecen al sector de servicios (Guercio et al., 2017), debido a que tienen una mayor concentración de activos fijos y utilizan estos como garantía frente a los créditos.

Sin embargo, entidades financieras no encuentran suficientemente adecuado este tipo de garantías y prefieren no realizar el préstamo y evitar impagos futuros (Motta, 2017). Guercio et al. (2017) en su estudio a pymes argentinas, agregan que las pymes pertenecientes al sector de alta tecnología también suelen presentar mayores limitaciones para la financiación, de las que ya presentan las pymes en general.

Con respecto al acceso al mercado de capitales, las pymes suelen tener mayores dificultades para cotizar en mercados de valores regulados, debido a los requisitos de admisión y las normas de divulgación presentes (Lardon y Deloof, 2014); también, existe un menor desarrollo de este sector en América Latina, y una asimetría de la información entre instituciones encargadas y empresas. La falta de políticas e incentivos para que las empresas accedan a este tipo de financiamiento, es una de las razones por las cuales países de la región tienen una participación muy baja en este mercado, en México solo el $12.90 \%$ de las pymes participan, siendo el porcentaje más alto en América Latina (Saavedra y Leticia, 2014).

Uno de los factores menos estudiados, corresponde al género de los directivos en las empresas; un estudio realizado en Argentina por Briozzo, Albanese y Santolíquido (2017), describe que el género de los altos cargos en las pymes es un factor influyente a la hora de tomar una decisión de financiación. Defienden que las mujeres suelen tener una mayor aversión al riesgo, y que estas prefieren tomar endeudamientos más altos a corto plazo, dado que existe una menor concentración de capital en sus empresas. Nikaido, Pais y 
Sarma (2015), mencionan que las empresas con mujeres propietarias y aquellas con industrias intensivas en capital, cuentan con mayores probabilidades de enfrentar limitaciones crediticias.

\subsection{Gestión de la planeación estratégica}

En primer lugar, es importante resaltar que la planeación no solo contribuye a mejorar la competitividad; sino que también permite que las diferentes áreas se armonicen y por tanto se realice un trabajo conjunto hacia un mismo objetivo (Mora, Vera y Melgarejo, 2015). Por su parte, Saldaña et al. (2014) describen la planeación estratégica como «una herramienta que permite a las organizaciones prepararse para enfrentar situaciones que se presentan en el futuro, ayudando con ello a orientar sus esfuerzos hacia metas realistas de desempeño» (p. 346).

Por lo tanto, es relevante que los responsables de las pymes estén conscientes de los factores claves dentro de su estrategia empresarial, para así dirigir mejor sus esfuerzos y finalmente dar cumplimiento a los objetivos planteados (Rangel, Aguilera y González, 2013). En palabras de Betancur, Yepes y Torres (2010), para que una empresa pueda tener éxito deberá contar con una adecuada planeación, según Argenti, citado por Masilo y Aguirre (2016), la falta de planeación empresarial es una de las causas del fracaso de los emprendimientos de las pymes mexicanas, en tanto que la toma de decisiones ineficiente, no permite alcanzar los objetivos propuestos, destruye valor y en un caso mayor genera quiebra de la empresa.

Por otra parte, García, Gálvez y Maldonado (2016), proponen que la elevada tasa de mortalidad de las empresas viene dada principalmente por una escasa aplicación de herramientas y métodos de análisis de información financiera.
Como plantean Saavedra y Espíndola (2016) «al no contar con un plan estratégico que incorpore los aspectos financieros del negocio difícilmente podrán alcanzar lo propuesto» (p. 9). Y, sumado a las situaciones anteriores, «muchas organizaciones no ven al proceso de administración estratégica como algo dinámico, cambiante y que debe ser revisado y ajustado permanentemente» (Quijano, Arguelles y Saui, 2015, p. 11).

Un ejemplo de lo anterior se evidencia en el estudio realizado en el Estado de Durango, México, Arrieta et al. (2015) señalan que la planeación que predomina en las pymes es de tipo informal, puesto que las empresas analizadas carecen de un proyecto de crecimiento que les permita tener una consolidación en el mercado. Adicionalmente, al analizar los diferentes estudios encontrados relacionados con este tema, las pymes que no implementan planes estratégicos, tienen una posición tecnológica débil, que viene acompañada de débiles habilidades administrativas de sus directores (Solano, Riascos y Aguilera, 2013).

Quijano, Arguelles, Magaña y Alcocer (2016), encontraron que a pesar de que el $70 \%$ de los fundadores de las empresas han obtenido títulos profesionales, estos no aplican la administración estratégica en sus empresas. Por su parte, Molina, López, y Schimtt (2016) exponen que «a la falta de planes estratégicos y operativos, el empresario no ha cambiado su forma tradicional de administrar la empresa, pues ha basado su toma de decisiones prácticamente en la intuición y la experiencia» (p. 103).

Saavedra, Tapia y Aguilar (2016), plantean que los empresarios en lugar de tomar decisiones con base en herramientas de análisis financiero, revisan de manera empírica algunas cuentas que parecen relevantes para ellos, sin el uso de una técnica financiera adecuada. Cabeza y Muñoz (2010) apoyan lo anterior, afirmando que en muchas ocasiones los empresarios 
toman decisiones apostando a la intuición. Acompañado de esto, se tiene una gran influencia de la familia, donde el componente emocional es fuerte y este puede llevar a una toma de decisiones inadecuada (Arenas y Rico, 2014).

Es así como, una información confiable, suficiente, oportuna y precisa, recobra relevancia, pues se podrá dar una toma de decisiones más sustentada (Restrepo y Vanegas, 2015). Tal como exponen Pacheco, Borda y Molina (2016), el uso de las finanzas gerenciales como parte esencial del funcionamiento, permitirá una toma de decisiones más acertada respecto a temas relacionados con inversión, ahorro, financiación, entre otros. Sin embargo, en el caso de México, descrito por Castro (2015), las pymes enfrentan un problema de planeación financiera, ya que los elementos que consideran para la toma de decisiones son empíricos y resultan ser insuficientes para desarrollar una planeación que les permita tener un crecimiento sostenible.

La responsabilidad social corporativa es un factor clave para generar diferenciación en la planeación estratégica; sin embargo, Nelling y Webb (2006), identifican que no hay una relación entre los gastos en responsabilidad social corporativa y un buen rendimiento; como se citó en Mousiolis, Zaridisb, Karamanisc y Rontogiannid (2014), la toma de decisiones está basada en sentimientos personales, influencias familiares o de algunos amigos. Y como indican Robles, Bocanegra y Ordocia (2015), «si los propietarios o gerentes de la mipyme no están preparados para efectuar sus actividades directivas de la manera correcta, se obstaculiza (...) la adecuada planeación estratégica» (p.110).

Por lo anterior, los tomadores de decisiones juegan un papel importante en la búsqueda de opciones de financiamiento (Rossi, Lombardi y Oliva, 2016). Además, desde la perspectiva de Xiang y Worthington (2015), la planeación estratégica es un eje fundamental para conocer una explicación sobre los motivos por los cuales las empresas buscan financiación. López, Maldonado, Pinzón y García (2016), agregan que las colaboraciones con otras empresas pueden impulsar el desarrollo de la innovación, pero para llegar a esto, los empresarios deben capacitar a sus empleados, no solo en investigación y desarrollo sino también, en el trabajo en equipo.

Adicional a lo anterior, es importante tener en cuenta el papel que tiene la planeación de la innovación para el crecimiento y desarrollo de las pymes, dado que de acuerdo a lo que plantea Rehman (2016), para acelerar la innovación en estas empresas, es necesario realizar una inversión en conocimiento sobre activos; además, como evidencia en un estudio en Chile, el alto costo de patentes e investigación y desarrollo, disminuye la posibilidad de las pymes de incrementar su desarrollo en innovación.

A continuación, se presenta de manera breve el efecto que tiene la falta de planeación estratégica en las pymes (Figura 4). 
Figura 4. Efectos de la planeación financiera

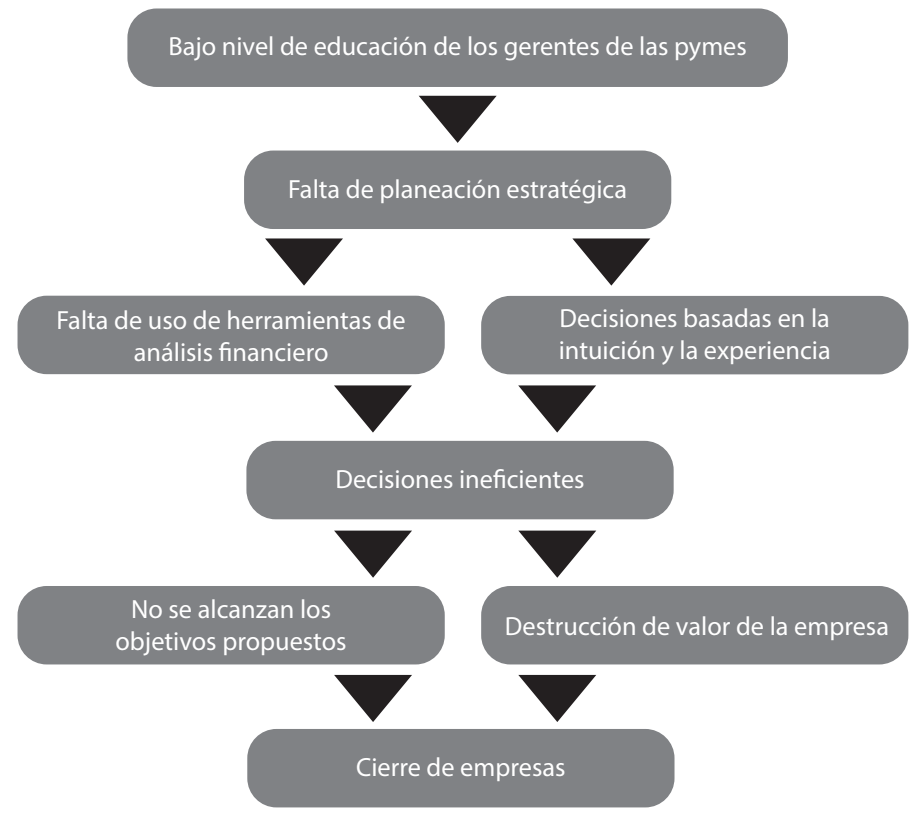

Fuente. Elaboración propia, basada en Molina et al., 2016; Masilo y Aguirre, 2016; Quijano et al., 2016; Saavedra y Espíndola, 2016; Saavedra et al., 2016.

\section{Conclusión}

$S^{c}$ e puede evidenciar que no existen muchos estudios que agrupen las problemáticas financieras que presentan las pymes en América Latina, en especial para el campo de estructura de capital. Sin embargo, la información encontrada, permite reflejar que existen factores en común que limitan el crecimiento $\mathrm{u}$ obstruyen la supervivencia de este segmento de empresas de la región latinoamericana.

Dentro de las principales problemáticas financieras encontradas, aparece el acceso al financiamiento, puesto que la banca tradicional impone el respaldo de garantías o el pago de altas tasas de interés. Dado esto, en países como México y Perú, existe una preferencia de apalancamiento por medio de recursos propios, frente al financiamiento externo; en otros casos como los de Ecuador y Costa Rica, los proveedores constituyen una atractiva alternativa de financiamiento. Y para el caso colombiano, a pesar de las restricciones para acceder a la banca comercial, esta sigue siendo la primera opción para los empresarios.

Adicional a ello, la asimetría de información entre instituciones financieras y pequeñas y medianas empresas, toma relevancia en el análisis de las limitaciones a la financiación. Otros factores influyentes, son los elevados costos financieros, el bajo desarrollo del mercado de capitales y del sistema financiero de la región, y los requerimientos elevados para la solicitud de créditos bancarios. 
Otro aspecto que obstaculiza el desarrollo y crecimiento de las pymes es la planeación estratégica, pues este tipo de empresa no la realiza de la manera más adecuada, principalmente debido a que los dirigentes de las pymes olvidan utilizar herramientas de análisis financiero, las cuales posiblemente les permitirían establecer una estrategia mejor para el direccionamiento de la empresa.

\section{Referencias}

Abor, J. Y. (2014). Bank finance and export activities of Small and Medium Enterprises. Review of Development Finance, 4(2014), 97- 103.

Alva, E. (2017). La desaparición de las microempresas en el Perú. Una aproximación a los factores que predisponen a su mortalidad. Caso del Cercado de Lima. Economía y Desarrollo, 158(2), 76-90.

Arenas, H. y Rico, D. (2014). La empresa familiar, el protocolo y la sucesión familiar. Estudios Gerenciales, 30, 252-258. doi: http://dx.doi.org/10.1016/j. estger.2014.02.013

Arrieta, D., Figueroa, E. G., Luna, J. E., Rivera, M. A., Meléndez, M. A., y Sotelo, J. G. (2015). La planeación estratégica en la innovación y permanencia de las MIPYMES. Revista Global de Negocios, 3(5), 1-14.

Avilés, F. (2017). Las pymes el motor de la economía sin fuente de gasolina. (Tesis de maestría). Recuperado de https://bit.ly/217M0oR

Banco Mundial. (2014). Facilitating SME financing through improved Credit Reporting. Report of the International Committee on Credit Reporting. Recuperado de https://bit.ly/2zRIbLG

Becicova, I. (2015). Is there a credit-gap in a periphery? The perception of this problem by small entrepreneurs. Journal of rural studies, 42, 11-20. Recuperado de https://bit.ly/2JCrZFx
Sumado a lo anterior, se encuentra que muchos de los directivos de este tipo de empresas tienen estudios enfocados a las finanzas muy básicos y que, por tanto, no conocen de cerca las oportunidades que podría brindarles bien sea el mercado financiero o el mercado de capitales, y así, superar de cierto modo su aversión al riesgo, ya que los recursos propios o los de familiares o amigos no serán suficientes para el crecimiento que quisiera tener la compañía.

Betancur, M., Yepes, A., y Torres, R. (2011). Riesgo de continuidad detectado a partir del análisis financiero en micro y pequeñas empresas de Medellín. Adversia, 9, 1-42.

Blancas. A., y López. C. (2014). Integración tecnológica y financiera de pequeñas y medianas empresas: hacia una nueva política de industrialización en México. Mexican Studies, 30(2), 522-556.

Blasco, A., Guaita. I., y Postiguillo. D. (2016). Risk Factors for SMEs Involved in Electric Power Feneration: Fluctuations in the discount rate (WACC). International journal of renewable energy research, 6(2), 680-686.

Botello, H. A. (2015). Determinants of access to credit for SME: evidence at the level of the firm in Latin America. Apuntes del CENES, 34(60), 247-276.

Brancati, E. (2015). Innovation financing and the role of relationship lending for SMEs. Small Bus Econ, 44 (2), 449-473. Recuperado de https://bit. ly/2HNs83r

Brenes, L. y Bermúdez, L. (2013). Condiciones actuales del financiamiento de las mipymes costarricenses. Tec. Empresarial, 7(2), 29-39. Recuperado http:// dx.doi.org/10.18845/te.v7i2.1511 
Briozzo. A., Albanese. D., y Santolíquido. D. (2017). Corporate governance, financing and gender: A study of SMEs from Argentinean Securities Markets. Contaduría y administración, 62(2), 358-376. https:// doi.org/10.1016/j.cya.2017.03.002

Briozzo, A., Fernández, A., Villar, L., Pesce, G. (2016). El rol de la banca pública en el financiamiento a Pymes. Estudio comparativo para la Argentina y Brasil. Revista Desarrollo y Sociedad, 76(2016), 205241.

Briozzo, A., Vigier, H., Castillo, N. Pesce, G., y Speroni, M. (2016). Decisiones de financiamiento en pymes: ¿Existen diferencias en función del tamaño y la forma legal? Estudios Gerenciales, 32(138), 71-81.

Cabeza, L., y Muñoz, A. E. (2010). Análisis del proceso de toma de decisiones, visión desde la pyme y la gran empresa de Barranquilla. Cuadernos Latinoamericanos de Administración, 6(10), 9-39.

Capa, L. B., Capa, X. R., \& Ollague, J. K. (2018). Estructura de capital en las pequeñas y medianas empresas bananeras de la provincia de El Oro. Universidad y Sociedad, 10(2), 294-303.

Castro, J. A. (2015). Diagnóstico administrativo y financiero de las micro, pequeñas y medianas empresas en Xicotepec de Juárez. Puebla. México D. F.: XX Congreso de contaduría administración e informática. Recuperado de https:// bit.ly/2lbVAr0

Córdova, M. S., Montalvo, C. M., y Bohórquez, N. (2014). Alternativa de financiamiento para pequeñas y medianas empresas familiares del sector comercial de Machala. Podium, 26, 27- 42.

Chiatchoua, C., y Castañeda, Y. (2015). Influencia de las mipymes en la generación de empleos en la región XI Texcoco, Estado de México (2000-2010). Quivera, 17(2), 55-71.

Dabla, E., Deng, Y., Ivanova, A., Karpowicz, I., Unsal, F., VanLeemput, E., y Wong, J. (2015). Inclusión financiera: un enfoque centrado en América Latina. Centro de Estudios Monetarios Latinoamericanos, 61(4), 244-281. Recuperado de https://bit.ly/2y8on97

Daskalakis. N., Eriotis. N., Thanou. E., y Vasiliou. D. (2014). Capital structure and size: new evidence across the broad spectrum of SME. Managerial Finance, 40(12), 1207-1222.
Domeher, D., Musah, G., y Hassan, N. (2017). Intersectorial Differences in the SME Financing Gap: Evidence from Selected Sectors in Ghana. Journal of African Business, 18(2), 194-220.

Duarte, L. G., Pastrana, I., Rosado, Y. L. (2015). La estructura financiera de las pymes en Yucatán de acuerdo a su antigüedad, tamaño y sector de actividad. Red Internacional de Investigadores en Competitividad Memoria del IX Congreso, 2248-2265. Recuperado de https://bit.ly/2MqQfID

Duréndez, A., Rocca, E. (2017). Factores determinantes que explican el acceso a la financiación bancaria: un estudio empírico en empresas peruanas. Small Business International Review, 1(1), 11-34.

Espejo, L., Robles, I., y Higuerey, A. (2017). Apalancamiento financiero en las empresas manufactureras de Ecuador. Revista Publicando, 4(13), 241-254.

Figueroa, J. (2014). Estudio de identificación y caracterización de brechas para el mejoramiento de la competitividad de la empresa pymes de Atacama en relación a la cartera de inversión de la región. (Tesis de pregrado). Recuperado de https://bit.ly/2JSn8iU

García, D., Gálvez, E., y Maldonado, G. (2016). Efecto de la innovación en el crecimiento y el desempeño de las mipymes de la Alianza del Pacífico. Estudios gerenciales, 33, 326-335. doi: http://dx.doi. org/10.1016/j.estger.2016.07.003

Guercio, M., Martínez, L., y Vigier, H. (2017). Las limitaciones al financiamiento bancario de las Pymes de alta tecnología. Estudios Gerenciales, 33(2017) 3-12. Recuperado de https://bit.ly/2t00RpN

Gutiérrez, M., Aguayo, P., y Panes, J. (2014). Estructura de financiamiento de las empresas chilenas aplicación del modelo de jerarquía. Horizontes Empresariales, 1, 7-19.

Jinjarak. Y., y Wingnaraja. G., (2016). An Empirical Assessment of the Export-Financial Constraint Relationship: How Different are Small and Medium Enterprises? World development, 79, 142-163. Recuperado de https://bit.ly/2JT1qLG

Kantis, H., Federico, J., Angelelli, P., y Ibarra, S., (2016). Business Performance in Young Latin American Firms. En M. Grazzi y C. Pietrobelli (Eds.), Firm Innovation and Productivity in Latin America and the Caribbean (pp. 167-207). New York, USA: Palgrave Macmillan. 
Kraus, A., y Litzenberger, R. H. (1973). A StatePreference Model of Optimal Financial Leverage. Journal of Finance, 28(4), 911-922.

Lardon, A., y Deloof, M. (2014). Financial disclosure by SMEs listed on a semi-regulated market: evidence from the Euronext Free Market. Financial disclosure by SME, 42, 361- 385.

Lecuona, R. (2014). Algunas lecciones de la experiencia reciente de financiamiento a las pymes Colombia, Costa Rica y México. CEPAL - Serie Financiamiento para el Desarrollo, (253), 2-37.

López, G. C., Maldonado, G., Pinzón, S. Y., y García, R. (2016). Colaboración y actividades de innovación en Pymes. Contaduría y Administración, 61, 56-58.

Mac an Bhairda, B. L. (2014). Culture's influences: An investigation of inter-country differences in capital structure. Borsa Istanbul Review, 14, 1-9.

Masilo, A., y Aguirre, M. (2016). Estudio de revisión sobre la planeación financiera y propuesta de modelo empírico para pymes de México. Revista CIMEXUS, 11(2), 73-105.

McGuinness, G., y Hogan, T. (2016). Bank credit and trade credit: Evidence from SME over the financial crisis. International Small Business Journal, 34(4), 412445.

Melgarejo, Z., Vera, M., y Mora, E. (2014). Diferencias de desempeño empresarial de pequeñas y medianas empresas clasificadas según la estructura de la propiedad del capital, caso colombiano. Suma de negocios, 5(12), 76-84.

Molina, R., López, A., y Schimtt, C. (2016). Factores que influyen en los problemas del emprendimiento de las mipymes: una percepción de los expertos. Revista Internacional Administración y Finanzas, 9(1), 95-113.

Mora, E. H., Vera, M., y Melgarejo, Z. A. (2015). Planificación estratégica y niveles de competitividad de las mipymes del sector comercio en Bogotá. Estudios gerenciales, 31, 79-87.

Mora, N., y Castillo, N. (2014). Evolución de las principales variables de acceso de las pymes al microcrédito en Colombia, 2002-mayo 2014. Magazín Empresarial, 10(24), 55-63.
Motta, V. (2017). Are SMEs in the hospitality industry less likely to experience credit constraint than other industries in the service sector? Evidence from Latin America. Tourism Economics, 1-21.

Mousiolis, D. T., Zaridisb, A. D., Karamanisc, K., y Rontogiannid, A. (2014). Corporate Social Responsibility in SME and MNE. The Different Strategic Decision Making. Procedia - Social and Behavioral Sciences, 175, 579-583.

Myers, S. C., y Majluf, N. S. (1984). Corporate financing and investment decisions when firms have information that investors do not have. Journal of Financial Economics, 13(2), 187-221.

Nelling, E., y Webb, E. (2006). Corporate social responsability and financial performance: the "virtuous circle" revisted. Review of cuantitative finance and accounting, 32 (2), 197-209.

Nikaido, Y., Pais, J., y Sarma, M. (2015). What Hinders and what enhances small enterprises access to formal credit in india? Review of Development Finance, $5,43-52$.

OECD. (2015). New Approaches to SME and Entrepreneurship Financing: Broadening the Range of Instruments. Recuperado de https://bit.ly/2vysODR

OCDE-ECLAC. (2012). Perspectivas económicas para América Latina 2013. Políticas de pymes para el cambio estructural. OECD Publishing.

Osano, H. M., y Languitone, H. (2016). Factors influencing access to finance by SMEs in Mozambique: case of SMEs in Maputo central business district. Journal of Innovation and Entrepreneurship.

Pacheco, F. A., Borda, L.M., y Molina, E.A. (2016). Caracterización socioeconómica para la gestión de las finanzas en pymes de Tunja, Boyacá. Vestigium Ire, 10(2), 147-165.

Pavón, L. (2016). Inclusión financiera de las pymes en el Ecuador y México. CEPAL - Serie Financiamiento para el Desarrollo (262), 1-82.

Pesce, G., Esandi, J. I., Briozzo, A. E., y Vigier, H. P. (2015). Decisiones de financiamiento en pymes: particularidades derivadas del entrelazamiento empresa-propietario. REGE- Revista de Gestão, $22(3), 357-380$. 
Prohorovs, A., Jakusonoka, I., y Beizitere, I. (2015). Is venture capital the source of financing for microenterprises? Proceedings of the 2015 International Conference Economic science for rural development, 39.

Quijano, R. A., Arguelles, L. A., Magaña D. E., y Alcocer, F. R. (2016). Diseño de estrategias y financiamiento en empresas familiares del sector camaronero de Altura de Campeche, México. Revista Global de Negocios, 4(2), 19-32.

Quijano, R. A., Arguelles, L. A., y Saui, J. A., (2015). Planeación estratégica y el control interno como promotores de rentabilidad en mipymes del sector turístico de la Ciudad de Campeche, México. Revista Iberoamericana para la Investigación y el Desarrollo Educativo, 10.

Rangel, J., Aguilera, L., y González, M. (2013). La innovación y el capital intelectual: factores de competitividad en la pyme manufacturera. Revistas Científicas, 14(2), 151-168.

Raufflet, E., Lozano, J., Barrera, E., y García, C. (2012). Responsabilidad Social Empresarial. Naucalpan de Juárez, México: Pearson Educación.

Rehman, N. (2016). Innovation performance of Chilean firms, a bivariate probit analysis. Journal of Entrepreneurship in Emerging Economies, 8, 1-24.

Restrepo, J., y Vanegas, J. (2015). Internacionalización de las pymes: análisis de recursos y capacidades internas mediante lógica difusa. Contaduría $y$ administración, 50, 836-863.

Rossi, M., Lombardi, R., y Oliva, N. (2016). The impact of corporate characteristics on the financial decisions of companies: evidence on funding decisions by Italian SMEs. Journal of Innovation and Entrepreneurship.

Ruiz, M. (2015). Fuentes y mecanismos de financiación en las pymes de Villavicencio Colombia. Revista Global de negocios 3, 93-110.
Saavedra, M. L., Tapia, B., y Aguilar, M. A. (2016). La gestión financiera en las pymes del Distrito Federal, México. Perspectiva Empresarial, 3, 55-69.

Saavedra, M., y Espíndola, G. (2016). El uso de la planeación financiera en las pyme de TI de México. Ciencias Administrativas, 15-31.

Saavedra, M. L., y Leticia, M. A., (2014). Alternativas de financiamiento para la micro, pequeña y mediana empresa latinoamericana. Revista Universitaria Ruta, 2.

Saavedra, M. L., y Tapia A.R. (2013). La problemática del financiamiento de la pyme en México: el caso de las sociedades financieras populares. Revista Visión Contable (11), 79-131.

Saldaña, C., García, G., Torres, G., Becerra, J., Martínez, D., y Muñoz, S. (2014). Diagnóstico para el desarrollo de una metodología en la toma de decisiones financieras para la micro y pequeña industria del estado de Tlaxcala. Ciencias Administrativas y Sociales, Handbook, 341-352.

Solano, O., Riascos, S., y Aguilera, A. (2013). Determinantes de los planes estratégicos de los sistemas de información en las pymes colombianas: caso Santiago de Cali, Colombia. Entramado, 9(1), 26-37.

Vera, M., Melgarejo, Z., y Mora, E. (2014). Acceso a la financiación en pymes colombianas: una mirada desde sus indicadores financieros. Revista innovar, 24, 148-160.

Xiang. D., y Worthington. A. (2015). Finance-seeking behaviour and outcomes for small- and mediumsized enterprises. International Journal of Managerial Finance, 11(4), 513-530.

Zevallos, E. (2003). Micro, pequeñas y medianas empresas en América Latina. Revista de la CEPAL 79, 53-70. 
